\title{
The detectability of habitable exomoons
}

\section{David M. Kipping, Stephen J. Fossey and Giammarco Campanella}

University College London, Dept. of Physics \& Astronomy, Gower Stree, London, N1 0XE, UK email: d.kipping@ucl.ac.uk

\begin{abstract}
Despite the number of known exoplanets increasing on an almost weekly basis, the question as to whether exoplanets host moons remains unanswered. Exomoons could be potential seats for life, as well as improving our understanding of planetary formation and celestial mechanics. Here we summarize our findings from an investigation into how detectable habitable-zone exomoons are with Kepler-class photometry.
\end{abstract}

\section{Introduction and methodology}

One of the principal goals of exoplanetary science is to discover whether habitable worlds, like the Earth, are common throughout the Galaxy. Indeed, determining the fraction of habitable planets around normal stars is the primary science goal of the Kepler Mission (Borucki et al. 2008). However, it has been proposed that moons could also be potentially common seats for life (Williams et al. 1997) and the task of detecting these bodies should be considered as pertinent as the search for a habitable planet.

Kipping (2009a, 2009b) proposed that exomoons could be unambiguously detected around transiting planets by coupling transit timing variations (TTV) and transit duration variations (TDV). The ratio of the two amplitudes provides both the mass and period of an exomoon; factors which influence the potential habitability. In this study (Kipping et al. 2009), we simulate the effect for a range of possible star-planet-moon configurations and generate the expected uncertainities using the noise properties of Kepler. We are then able to evaluate the range of habitable-zone exomoons which may be detectable with Kepler, or photometry of equal quality.

\section{Summary of results}

- Exomoons are most easily detected around low-density exoplanets, because both the planetary transit signal and the perturbation due to an exomoon are maximal.

- Habitable-zone exomoons down to $0.2 \mathrm{M}_{\oplus}$ may be detected around the optimum targets (based on a $0.4 \mathrm{M}_{\odot} \mathrm{M}$-dwarf at 10pc).

- Dynamically stable $1 \mathrm{M}_{\oplus}$ exomoons may be surveyed for up to $200 \mathrm{pc}$ away, consituting 25,000 stars within Kepler's field of view.

\section{References}

Borucki, W. J. et al. 2008, in: M. Livio, K. Sahu \& J. Valenti (eds.), A Decade of Extrasolar Planets around Normal Stars, (Cambridge: Cambridge University Press), p. 36

Kipping, D. M. 2009a, MNRAS 392, 181

Kipping, D. M. 2009b, MNRAS 396, 1797

Kipping, D. M., Fossey, S. J., \& Campanella, G. 2009, MNRAS, in print

Williams, D. M., Kasting, J. F., \& Wade, R. A. 1997, Nature 385, 235 